\title{
SEGNET-BASED EXTRACTION OF WETLAND VEGETATION INFORMATION FROM UAV IMAGES
}

\author{
Tang Tingyuan ${ }^{1}, \mathrm{Fu}$ Bolin $^{*}$, Lou Peiqing ${ }^{1}, \mathrm{Bi} \mathrm{Lu}^{1}$ \\ ${ }^{1}$ Guilin University of Technology, 541006, Guangxi - TangtyRS@126.com
}

KEY WORDS: Multi-class SegNet model, Fusion SegNet model, wetland vegetation, extraction multi-classification, UAV

\begin{abstract}
:
This study takes Guangxi Huixian National Wetland Park as the research area, and uses the UAV image and ground measured tag data as the data source. The SegNet model is used to extract the wetland vegetation information in the study area, further verification multiple classification SegNet model and fusion multiple SegNet model of single/double classification precision of the two ways of extracting karst wetland vegetation information. The experimental results show that the Kappa coefficient of the multi-segmented SegNet model is 0.68 , while the multi-class SegNet model has a classification effect of 0.59 . The classification effect of the karst wetland vegetation information extracted by multiple single/double-class SegNet models is more than the multi-classification. The SegNet model has high precision.
\end{abstract}

\section{INTRODUCTION}

At present, the extraction of wetland vegetation information is mainly based on machine learning algorithm. Among the machine learning algorithm, random forest algorithm ${ }^{[1]}$ and decision tree ${ }^{[2-3]}$ algorithm have good effects on the extraction of wetland vegetation information. Zhu, Junfeng ${ }^{[1]}$ applied the random forests, a machine learning method, to automatically separate sinkholes from other depressions in a karst region in central Kentucky. The sinkhole-extraction random forest was grown on a training dataset built from an area where LiDARderived depressions were manually classified through a visual inspection and field verification process. The weighted random forest achieved an average accuracy of $89.95 \%$ for the training dataset, demonstrating that the random forest can be an effective sinkhole classifier. Testing of the random forest in another area, however, resulted in moderate success with an average accuracy rate of $73.96 \%$. In short, the current machine learning algorithm has good precision for wetland vegetation information extraction, but the deep learning algorithm is rarely used in this field. Pengbin Zhang ${ }^{[4]}$ propose the novel multiscale deep learning models, namely ASPP-Unet and ResASPPUnet are proposed for urban land cover classification based on very high resolution (VHR) satellite imagery. It is shown that the ResASPP-Unet model with 11 layers and 64 IFMs based on 8-band WV2 imagery produced the highest classification accuracy $(87.1 \%$ for WV2 imagery and $84.0 \%$ for WV3 imagery). In summary, deep learning algorithm is mainly applied to the extraction of single category of architectural information based on spaceborne optical images with an accuracy of more than $80 \%$, while deep learning algorithm based on multi-category information extraction of low-altitude man-machine image wetlands is rarely studied.

This paper takes the core area of Guilin Huizhou Karst Country Wetland Park in Guangxi as the research area, and uses the low-altitude drone image and measured tag data as the data source. Based on SegNet algorithm, the model for extracting vegetation information of karst wetland is constructed, with accuracy and recall rate. The F1-score and Kappa coefficients are used to evaluate the accuracy of the model. The accuracy of the karst wetland vegetation information extracted from the multi-class SegNet model and the fusion of multiple single/double SegNet models is further verified.

\section{STUDY AREA AND DATA}

\subsection{Overview of the Study Area}

The core area of Huixian Karst National Wetland Park in Guilin, Guangxi is the research area. The core area is the area with the least vegetation damage and the most complete preservation of vegetation information. which is distributed with lotus flowers, karst herbs, water hyacinth, bamboo, lindens and other vegetation ${ }^{[5-6]}$, as shown in figure 1 .

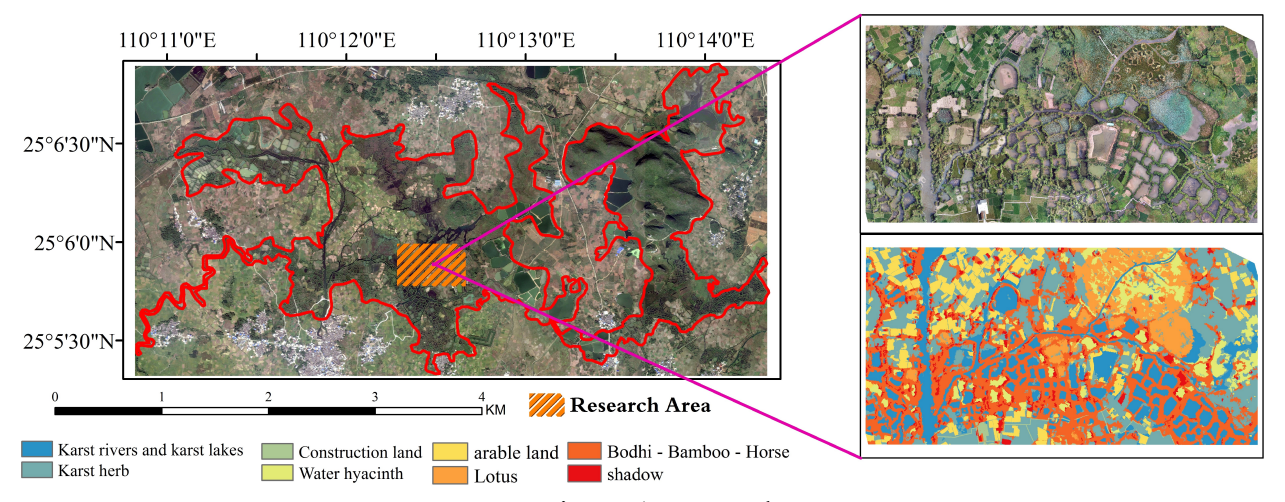

Figure 1 Research Area

\footnotetext{
*Fu Bolin - E-mail:fbl2012@126.com

Tang Tingyuan - E-mail:TangtyRS@126.com
} 


\subsection{Data}

The data used in this paper is processed low-altitude drone aerial photography and measured tag data. The drone data is collected by the DJI Dajiang Phantom 4 Pro light and small drone with 20 million effective pixels. The collection time is July 13-14, 2018. The weather is clear and cloudless, and the flying height of the drone is $86 \mathrm{~m}$., flying 1 sorties. The test label data was obtained from the wetland vegetation type survey, and the survey time was synchronized with the image capture time. The tag data contains eight feature categories, namely karst river-karst lake, karst herb, cultivated land, water hyacinth, construction land, linden-bamboo-matrix, lotus and shadow.

\section{RESEARCH METHODS}

\subsection{SegNet}

The SegNet model of this study consists of 26 layers of Convolutional, 5 layers of MaxPooling [7], 5 layers of upsampling and 1 layer of Softmax. Each layer has a Kernel size of $3 * 3$, a Stride of 1 , and an activation function of Relu. Padding is the same type, and the number of convolution kernels is set to $64,128,256$, and 512, respectively. Each layer has a layer of batch normalization after convolution; the pooling kernel size of each layer is $2 * 2$, and the sliding step size is 1 . The edge padding is the same type; the sampling of each layer is set to a row and column magnification of 2 , and each layer is sampled and has a feature in a merge layer fusion encoder; the last layer is Softmax, and the number of nodes is set to type. Quantity, the probability that each pixel belongs to each category, and the model is shown in the SegNet network in Figure 2.

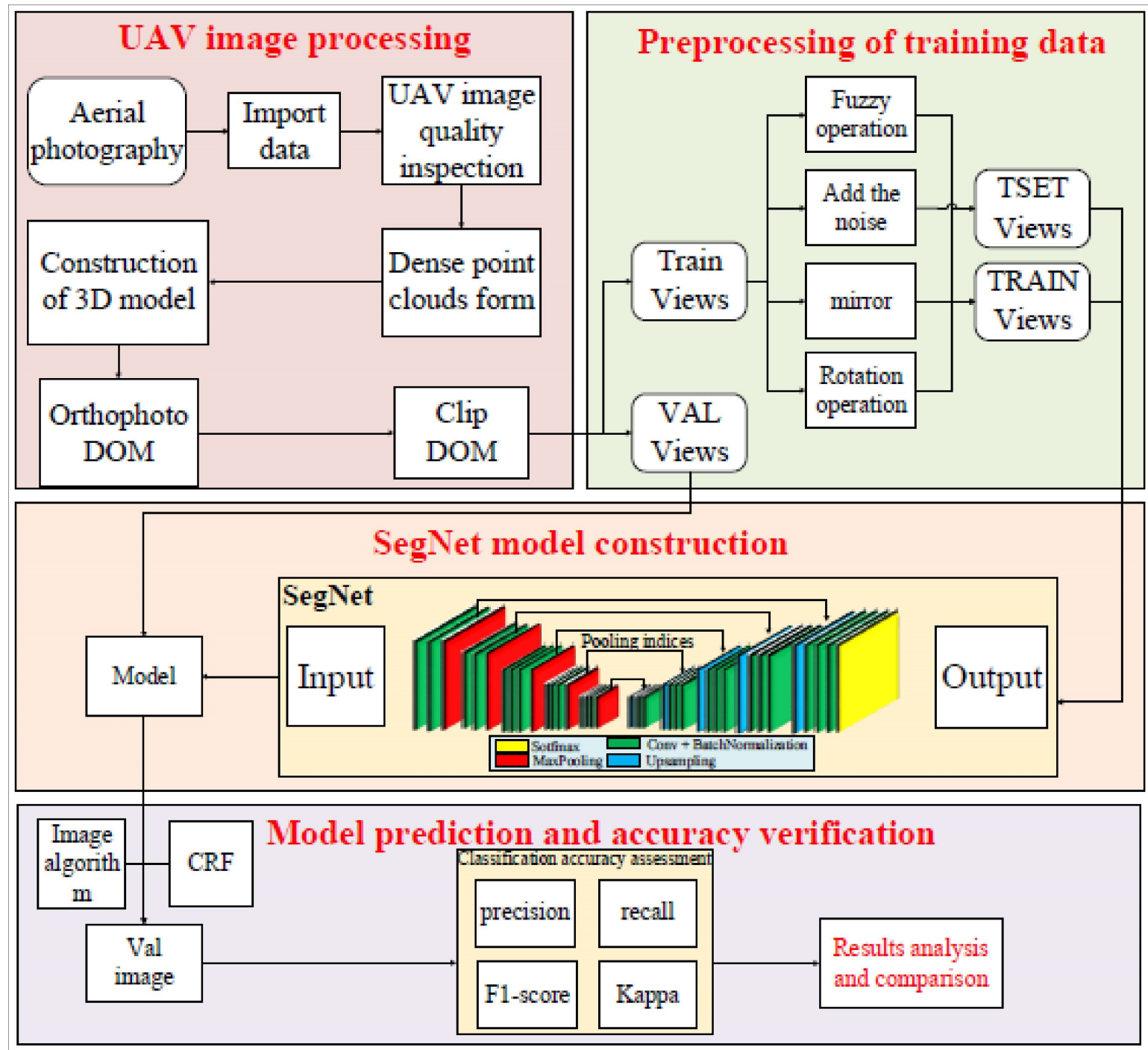

Figure 2 technical flow char

\subsection{SegNet Model Construction}

This study investigates SegNet algorithm to extract the lowaltitude uav image classification accuracy and SegNet karst wetland vegetation information model and the fusion of multiple SegNet single/double classification model two ways respectively karst wetland vegetation information extraction accuracy difference, in this paper, the general technical route as shown in figure 2, mainly including the training data preprocessing, SegNet model building, model prediction and accuracy evaluation of three steps. 


\subsubsection{Preprocessing of Training Data}

The training data preprocessing is to randomly cut the processed training data set into 100,000 image sets with a size of $256 * 256$, and perform data enhancement operations on the cut image set, wherein $75 \%$ of the 100,000 image sets are model training data, $25 \%$ is the model test data. The data enhancement operation process is as follows: 1 image random rotation $90^{\circ}, 180^{\circ}, 270^{\circ} ; 2$ image mirroring operation along the y axis; 3 image Gaussian blur operation; 4 random addition noise operation (gauss noise, salt and pepper noise).

\subsubsection{SegNet Model Construction}

The pre-processed training data set is input into the SegNet model, and the optimal SegNet model is obtained by iteratively updating the weight parameters of each layer of the model through the training data set. Due to the limited performance of

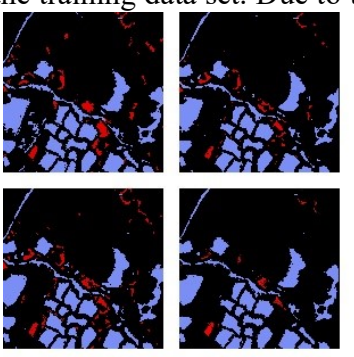

Karst rivers and karst lakes shadow
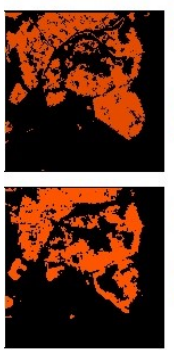

Lotus

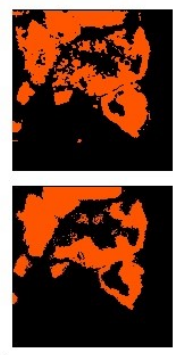

otus

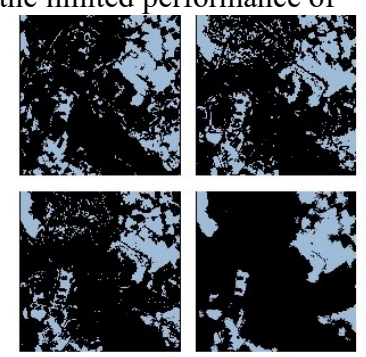

Karst herb

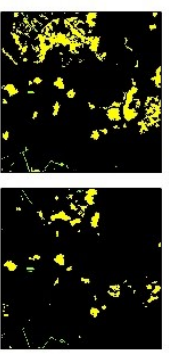

Water hyacinth Construction land
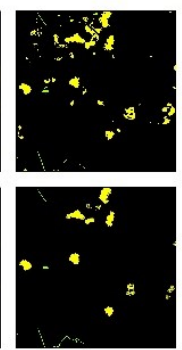

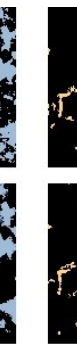
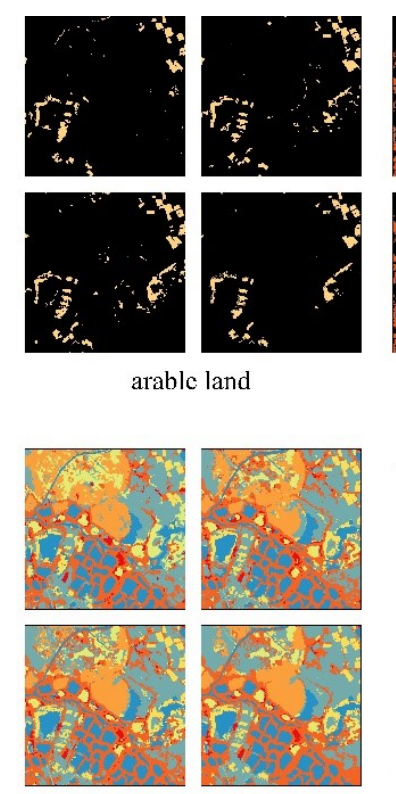

Multiple classification

the currently used desktop terminal, the batch parameter size (Batch-size) is set to 8; and since most of the models are multiclassification models, the model classification achieves better results, and the model optimizer algorithm (Optimizer) is set ${ }^{[8]}$ for SGD, loss function (Loss Function) is categorical_crossentropy loss; in order to compare the influence of parameter period $(\mathrm{EPOCH})$ on the classification accuracy of the model, set the parameter period (EPOCH) to 5 and 10 for each image set of size $256^{*} 256$. The remaining training data set setting parameter period (EPOCH) is 10 .

\subsubsection{Model Prediction and Accuracy Verification}

The trained model predicts the verification image set, but in order to improve the classification effect, the image algorithm and the conditional random field algorithm are added to postprocess the image prediction values. Finally, the results of each model classification are shown in Figure 3.

arable land

Bodhi - Bamboo - IIorse

Figure 3 SegNet model classification results with different training parameters EPOCH and whether to join CRF (training image size is $256^{*} 256$ )

To contrast classification SegNet SegNet model of multiple single/double classification model and the fusion precision of the two ways of extracting karst wetland feature information differences, around the sample size of content types, according to the study area weighted fusion multiple training data set the image size is $256 * 256$ forecast model of single/double classification of the image, get the classification results fusion model.

This study compared the classification results of the model with the ground truth data, and compared and analyzed precision indicators such as precision, recall, f1-score [9], Kappa coefficient ${ }^{[10]}$, as shown in table 2,3 and 4.

\section{RESULTS ANALYSIS AND ACCURACY VERIFICATION 4 RESULTS}

\subsection{Multi-class SegNet Model}

The classification effect of karst wetland vegetation information extracted by multi-class SegNet model is analyzed
(Fig. 3). The accuracy of the model image classification results with different training parameters is verified by the real label collected in the field, and the Kappa coefficient and accuracy rate are calculated. , recall rate, F1-score four types of accuracy indicators (Table 1) to assess the classification accuracy of the model. The accuracy indexes of the models trained by three different parameters EPOCH are close. The highest Kappa coefficient is the training parameter EPOCH is 10 and the model added to the CRF algorithm is 0.61 . The lowest is the model with the training parameter EPOCH of 5, which is 0.59 . The highest of the accuracy indicators F1-score is the model with the training parameter EPOCH of 5 and the training parameter EPOCH of 10 and the model with the CRF algorithm, which is 0.65 ; the lowest is the model with the training parameter EPOCH of 5, which is 0.62. Combining the accuracy indexes of the three models, the training parameters EPOCH is 10, and the model with the CRF algorithm is the best to extract the karst wetland vegetation types. The other two models are close to the model classification effect.

In summary, the multi-class SegNet model extracts the karst wetland vegetation types, the model Kappa coefficient and F1- 
score of the three different training parameters are both around 0.6; compared with the other two models, the training parameters $\mathrm{EPOCH}$ is 10 and the model added to the CRF algorithm The classification works best. Due to the phenomenon of remote sensing images with the same spectrum of foreign objects, type misclassification affects the classification accuracy of the model.

\begin{tabular}{|c|c|c|c|c|c|c|}
\hline & & parameter settings & precision & recall & F1-score & Kappa \\
\hline \multirow{6}{*}{ Model 1} & \multirow{3}{*}{ Karst rivers and karst lakes } & $\mathrm{EPOCH}=5$ & 0.92 & 0.86 & 0.89 & 0.79 \\
\hline & & $\mathrm{EPOCH}=10$ & 0.93 & 0.86 & 0.90 & 0.79 \\
\hline & & $\mathrm{EPOCH}=10 \& \mathrm{CRF}$ & 0.94 & 0.89 & 0.91 & 0.80 \\
\hline & \multirow{3}{*}{ shadow } & $\mathrm{EPOCH}=5$ & 0.38 & 0.40 & 0.39 & 0.79 \\
\hline & & $\mathrm{EPOCH}=10$ & 0.37 & 0.55 & 0.44 & 0.79 \\
\hline & & $\mathrm{EPOCH}=10 \& \mathrm{CRF}$ & 0.27 & 0.21 & 0.24 & 0.80 \\
\hline \multirow{3}{*}{ Model 2} & \multirow{3}{*}{ Karst herb } & $\mathrm{EPOCH}=5$ & 0.61 & 0.65 & 0.63 & 0.50 \\
\hline & & $\mathrm{EPOCH}=10$ & 0.66 & 0.62 & 0.64 & 0.52 \\
\hline & & $\mathrm{EPOCH}=10 \& \mathrm{CRF}$ & 0.76 & 0.61 & 0.68 & 0.58 \\
\hline \multirow{3}{*}{ Model 3} & \multirow{3}{*}{ arable land } & $\mathrm{EPOCH}=5$ & 0.54 & 0.71 & 0.61 & 0.59 \\
\hline & & $\mathrm{EPOCH}=10$ & 0.49 & 0.70 & 0.58 & 0.56 \\
\hline & & $\mathrm{EPOCH}=10 \& \mathrm{CRF}$ & 0.60 & 0.68 & 0.63 & 0.62 \\
\hline \multirow{3}{*}{ Model 4} & \multirow{3}{*}{ Bodhi - Bamboo - Horse } & $\mathrm{EPOCH}=5$ & 0.68 & 0.78 & 0.73 & 0.63 \\
\hline & & $\mathrm{EPOCH}=10$ & 0.65 & 0.79 & 0.71 & 0.61 \\
\hline & & $\mathrm{EPOCH}=10 \& \mathrm{CRF}$ & 0.75 & 0.71 & 0.73 & 0.65 \\
\hline \multirow{3}{*}{ Model 5} & \multirow{3}{*}{ Lotus } & $\mathrm{EPOCH}=5$ & 0.79 & 0.82 & 0.81 & 0.77 \\
\hline & & $\mathrm{EPOCH}=10$ & 0.79 & 0.73 & 0.76 & 0.71 \\
\hline & & $\mathrm{EPOCH}=10 \& \mathrm{CRF}$ & 0.79 & 0.75 & 0.77 & 0.73 \\
\hline \multirow{6}{*}{ Model 6} & \multirow{3}{*}{ Water hyacinth } & $\mathrm{EPOCH}=5$ & 0.69 & 0.35 & 0.47 & 0.41 \\
\hline & & $\mathrm{EPOCH}=10$ & 0.72 & 0.50 & 0.59 & 0.41 \\
\hline & & $\mathrm{EPOCH}=10 \& \mathrm{CRF}$ & 0.96 & 0.49 & 0.65 & 0.29 \\
\hline & \multirow{3}{*}{ Construction land } & $\mathrm{EPOCH}=5$ & 0.83 & 0.30 & 0.44 & 0.41 \\
\hline & & $\mathrm{EPOCH}=10$ & 0.83 & 0.29 & 0.43 & 0.41 \\
\hline & & $\mathrm{EPOCH}=10 \& \mathrm{CRF}$ & 0.91 & 0.18 & 0.30 & 0.29 \\
\hline \multirow[t]{3}{*}{ Model all } & \multirow{3}{*}{ Multiple classification } & $\mathrm{EPOCH}=5$ & 0.68 & 0.66 & 0.65 & 0.60 \\
\hline & & $\mathrm{EPOCH}=10$ & 0.67 & 0.62 & 0.62 & 0.59 \\
\hline & & $\mathrm{EPOCH}=10 \& \mathrm{CRF}$ & 0.73 & 0.62 & 0.65 & 0.61 \\
\hline
\end{tabular}

Table 1: EPOCH is different, whether to join the CRF model classification results accuracy table

\subsection{Single/Double Classification SegNet Model}

By visually interpreting multiple single/double-class SegNet models to extract the classification effect of karst wetland vegetation information (Fig. 3), combined with Kappa coefficient and accuracy, recall rate, F1-score four types of precision indicators (Tables 1) ), found as follows:

(1) Among the karst wetland-karst lake and shadow double classification types, the model classification effect of three training parameters is obviously better. The training parameters EPOCH of Kappa coefficient is 10 and the model added with CRF algorithm is higher than the other two models. It is 0.80 . Among the karst river-karst lake types, the highest accuracy of F1-score is that the training parameter EPOCH is 10 and the model with CRF algorithm is 0.91 , and the lowest is 0.89 for the training parameter EPOCH of 5. Among the shadow types, the model F1-score with the training parameter EPOCH of 10 is the highest, which is 0.44 ; the training parameter EPOCH is 10 , and the model F1-score with the CRF algorithm is the lowest, which is 0.24 .

(2) Among the karst herb single classification types, the highest Kappa coefficient and F1-score accuracy are the training parameters EPOCH 10 , the training image size is $256^{*} 256$, and the model added to the CRF algorithm is $0.68,0.58$, respectively. The training parameter $\mathrm{EPOCH}$ is 5, and the model with the training image size of $256 * 256$ is 0.05 and 0.08 higher. Comparing the models with the same training parameters and different training images, the F1-score accuracy and Kappa coefficient of the model with the training image size of $128^{*} 128$ are the lowest, and the accuracy and effect of the other models are close.

(3) Among the single classification types of cultivated land, the training parameters EPOCH is 10 , the training image size is $128 * 128$, and the model Kappa coefficient and F1-score with the $\mathrm{CRF}$ algorithm are the highest, $0.65,0.64$. Other models are close to it, but training The parameter EPOCH is 10, the Kappa coefficient of the model 0.45 with the training image size of 
96*96, and the F1-score of 0.42 have the lowest precision.

(4) In the single classification type of linden-bamboo-matrix, the Kappa coefficient and F1-score precision of each model are close. The highest Kappa coefficient is 10 for the training parameter EPOCH and 0.67 for the CRF algorithm. The lowest is the training parameter $\mathrm{EPOCH}$ is 10 ; the highest $\mathrm{F} 1$-score is the training parameter EPOCH is 10 and the model with the CRF algorithm is 0.75 , the lowest The training parameter $\mathrm{EPOCH}$ is 10 .

(5) For the single classification type of lotus flower, the model with the training parameter EPOCH of 5 is the best, with Kappa coefficient of 0.77 and F1-score accuracy of 0.81 ; followed by training model EPOCH of 10 and training image size of $256^{*} 256$, Kappa The coefficient is 0.73 and the F1score accuracy is 0.77 . The lowest is the model with the training parameter EPOCH of 10. The Kappa coefficient is 0.71 and the F1-score accuracy is 0.76 .

(6) Water hyacinth and construction land double classification type. The model with training parameter EPOCH of 10 has the lowest Kappa coefficient of 0.29 , and the other two models have the highest Kappa coefficient of 0.41. Among the construction land types, the F1-score accuracy of the model 0.44 with the training parameter EPOCH of 5 is 0.14 higher than the training parameter EPOCH of 10 and the F1-score of the model 0.30 added to the CRF algorithm. Among the water hyacinth types, the training parameter $\mathrm{EPOCH}$ is 10 and the model with the CRF algorithm is 0.65 , and the f1-score has the highest accuracy. The training parameter EPOCH is 5, and the F1-score of the model 0.47 has the lowest accuracy.

In the classification of karst wetland vegetation information extracted by single/double classification SegNet model, the following conclusions are drawn: 1 wetland-karst lake type, karst herb type, cultivated land type, linden-bamboo-matrix with large sample size and large area Type and water hyacinth type, with the increase of training parameters EPOCH and the addition of CRF algorithm, will improve the classification accuracy of the model; 2 the size of the model training image has little effect on the accuracy of extracting the vegetation type of karst wetland; 3 CRF algorithm will be added Eliminating the type of sample area is small, resulting in a decrease in the accuracy of the model with more sample area.

\subsection{Multi-class SegNet Model and Comparison of Classification Accuracy of Multiple Single/Double-Class SegNet Models}

Multi-classification model and merging multiple single/double classification models (fusion model) classification results (Figure 3.), using real label data to calculate the Kappa coefficient and accuracy rate, recall rate, F1-score four types of precision indicators (Table 2) Evaluate the accuracy of the two models. Compared with the accuracy of each type of classification model, the F1-score accuracy of each type of fusion model is improved, and the type of cultivated land and lotus type are improved by 0.19 . Kappa coefficient of fusion model 0.68 , macro-average (macro avg) of 0.79 of multiclassification model The Kappa coefficient of 0.59 , the microaverage of 0.67 , the macro-average of 0.67 , and the weighted average of 0.71 are higher than 0.07 , and 0.06 , respectively, indicating that the classification effect of extracting karst wetland vegetation information by combining multiple single/double-class SegNet models is Multi-class SegNet model is good.

\begin{tabular}{c|ccc|ccc}
\hline & \multicolumn{3}{|c|}{ Multi-classification model } & \multicolumn{3}{c}{$\begin{array}{c}\text { Fusion of multiple single/double } \\
\text { classification models }\end{array}$} \\
\cline { 2 - 7 } & precision & recall & F1-score & precision & recall & F1-score \\
\hline Karst rivers and karst lakes & 0.91 & 0.86 & 0.88 & $\mathbf{0 . 9 2}$ & $\mathbf{0 . 8 9}$ & $\mathbf{0 . 9 1}$ \\
Karst herb & 0.58 & $\mathbf{0 . 6 6}$ & 0.62 & $\mathbf{0 . 8 1}$ & 0.60 & $\mathbf{0 . 6 9}$ \\
Water hyacinth & 0.58 & $\mathbf{0 . 5 2}$ & 0.55 & $\mathbf{0 . 9 6}$ & 0.41 & $\mathbf{0 . 5 7}$ \\
Construction land & $\mathbf{0 . 8 2}$ & 0.34 & 0.48 & 0.42 & $\mathbf{0 . 6 9}$ & $\mathbf{0 . 5 2}$ \\
arable land & 0.39 & 0.70 & 0.50 & $\mathbf{0 . 6 6}$ & $\mathbf{0 . 7 2}$ & $\mathbf{0 . 6 9}$ \\
Lotus & $\mathbf{0 . 8 5}$ & 0.51 & 0.64 & 0.81 & $\mathbf{0 . 8 6}$ & $\mathbf{0 . 8 3}$ \\
Linden - Bamboo - Horse & 0.63 & $\mathbf{0 . 8 3}$ & 0.72 & $\mathbf{0 . 7 4}$ & 0.77 & $\mathbf{0 . 7 5}$ \\
shadow & 0.62 & $\mathbf{0 . 5 2}$ & 0.57 & $\mathbf{0 . 9 6}$ & 0.46 & $\mathbf{0 . 6 2}$ \\
\hline Kappa & \multicolumn{3}{|c|}{0.59} & & $\mathbf{0 . 6 8}$ & $\mathbf{0 . 7 9}$ \\
macro avg & & 0.67 & & & & \\
\hline
\end{tabular}

Table 2 Comparison of prediction results of multi-classification model and fusion single/double classification model

\section{CONCLUSIONS AND PROSPECTS}

In this study, the low-altitude drone image and measured tag data are used as the data source. The core area of Huixian National Wetland Park in Guilin, Guangxi is taken as the research area. The SegNet algorithm is used to extract the vegetation information of the drone image karst wetland and explore the high resolution of SegNet extraction. The accuracy of image information of karst wetland vegetation, as well as the classification of karst wetland vegetation types by multi-class SegNet model and fusion single/double classification SegNet model, the following conclusions are drawn: (1) SegNet model extracts low-altitude drone image karst wetland multi-species vegetation The Kappa coefficient is 0.59 and the macro avg is
0.67. The extraction of karst rivers and karst lakes is the best, F1-score is as high as 0.88 , while the effect of extracting water hyacinth is the worst, F1-score is 0.55 . (2) The classification effect of extracting karst wetland vegetation information by combining multiple single/double classification SegNet models is higher than that of multi-class SegNet model. The Kappa coefficient of fusion model is 0.68 , and the higher classification model is 0.09 higher. Compared with multi-classification model, each vegetation type Accuracy, the F1-score of each vegetation type in the fusion model has been improved, and the most improved is the lotus, which is increased from 0.64 to 0.83 .

This study applies SegNet to the extraction classification of 
karst wetland vegetation types, and there are some shortcomings, which will be improved one by one in future work research. The model classification of karst herb type, water hyacinth type, linden-bamboo-matrix type is low. Considering the spectral features and texture features of three types of ground objects, DSM images, texture features and geometry are added to the training SegNet model. Information such as features to discuss whether the accuracy of extracting vegetation types from karst wetlands can be improved.

\section{ACKNOWLEDGEMENTS}

Thanks to mentor Fu Bolin and the colleagues in the laboratory for their help and my parents for their support. It is everyone's credit that I can successfully complete this experiment and thesis.

\section{REFERENCES}

[1] Zhu J, Pierskalla W P, 2016. Applying a weighted random forests method to extract karst sinkholes from LiDAR data. Journal of Hydrology, 533:343352.doi.org/10.1016/j.jhydrol.2015.12.012.

[2] Zhang Yingying, Cai Xiaobin, Song Xinxin, .etc., 2018. Remote Sensing Information Extraction of Hydrophyte in Honghu Lake based on Decision Tree. Wetland Science, 16(02):213-222.

[3] Lu C , Huaqiang D U, Guomo Z, et al. Combination of decision tree and mixed pixel decomposition for extracting bamboo forest information in China[J]. Journal of Remote Sensing, 2019.

[4] Pengbin Zhang, Yinghai Ke, Zhenxin Zhang, 2018. Urban Land Use and Land Cover Classification Using Novel Deep Learning Models Based on High Spatial Resolution Satellite Imagery. Sensors, 18(11), 3717.doi: $10.3390 / \mathrm{s} 18113717$
[5] Guang-Ping, X. U. , Yan-Qiong, L. I. , Yu-Yi, S. , DeNan, Z. , Ying-Jie, S. , \& Zhong-Feng, Z. , et al. (2019). Soil organic carbon distribution and components in different plant communities along a water table gradient in the huixian karst wetland in guilin. Environmental Science.

[6] Feng, W. . (2017). Fish diversity in huixian wetland in guangxi. Wetland Science, 15(2), 256-262.doi: 10.13248/j.cnki.wetlandsci.2017.02.014

[7] Yang Jianyu,Zhou Zhenxu,Du Zhenrong,Xu Quanquan,Yin Hang,Liu Rui.Rural construction land extraction from high spatial resolution remote sensing image based on SegNet semantic segmentation model[J].Transactions of the Chinese Society of Agricultural Engineering (Transactions of the CSAE),2019,35(5):251-258.doi:10.11975/j.issn.1002-6819. 2019.05.031

[8] Glorot X , Bordes A, Bengio Y, 2010. Deep Sparse Rectifier Neural Networks. Proceedings of the 14th International Conference on Artificial Intelligence and Statistics (AISTATS).

[9] Yanna Wang,Cunzhao Shi,Baihua Xiao,Chunheng Wang,Chengzuo Qi, 2018. CRF based text detection for natural scene images using convolutional neural network and context information[J]. Neurocomputing,295.doi:10.1016/j.neucom.2017.12.058

[10] Zhang Q S, Xu B L, Liu L D, et al., 2012. Analysis of genetic diversity among ChinesePleurotus citrinopileatusSinger cultivars using two molecular marker systems (ISSRs and SRAPs) and morphological traits[J].World Journal of Microbiology \& Biotechnology, 28(5):2237-2248.doi:10.1007/s11274-012-1031-4 